\title{
Evaluation of antileishmanial potential of Gentiana kurroo Royle by in vitro and in silico methods
}

\author{
Arushdeep Sidana $^{1,2^{*}}$, Shiwani Kaushal ${ }^{3,4}$, Umar Farooq $^{1}$ \\ ${ }^{1}$ Molecular and Immuno-parasitology Laboratory, Faculty of Applied Sciences and Biotechnology, Shoolini University, Solan-173229 HP, India. \\ ${ }^{2}$ School of Biological and Paramedical Sciences, RIMT University, Punjab-147301, India. \\ ${ }^{3}$ Department of Biosciences, Asian Educational Institute, Sirhind Road, Patiala, Punjab-147001, India. \\ ${ }^{4}$ Plant Tissue Culture Laboratory, Faculty of Applied Sciences and Biotechnology, Shoolini University, Solan-173229 HP, India.
}

\section{ARTICLE INFO \\ Article history: \\ Received on: 25/06/2017 \\ Accepted on: 10/11/2017 \\ Available online: 27/02/2018}

Key words:

Antileishmanial, Gentiana

kurroo, in silico docking,

Leishmania donovani.

\begin{tabular}{l}
\hline ABSTRACT \\
The objective of the present investigation was to evaluate the antileishmanial efficacy of Gentiana kurroo Royle by in \\
vitro assay and in silico molecular docking method. In vitro antileishmanial screening of four different concentrations \\
of the methanolic root extract of $G$. kurroo was carried out against promastigotes of Leishmania donovani. After in \\
vitro evaluation, a total of 13 already known phyto-constituents present in the methanolic root extract of $G$. kurroo \\
were docked in silico with five reported drug targets of $L$. donovani to evaluate their binding and inhibitory potential \\
and to identify the active constituent responsible for antileishmanial activity. The methanolic extract of $G$. kurroo \\
roots at a dose of $500 \mu \mathrm{g} / \mathrm{ml}$ showed $43.1 \%$ inhibition of Leishmania promastigotes after incubation for 24 hours at \\
$25^{\circ} \mathrm{C}$. In silico docking analysis of 13 phyto-constituents present in the roots of $G$. kurroo revealed that gentioside had \\
maximum estimated binding and inhibitory capacity for most of the drug targets followed by norswertianolin. The \\
findings of the present study suggested that these two compounds may be the major players responsible for the in vitro \\
antileishmanial activity and therefore, should be further evaluated to establish their antileishmanial potential against \\
amastigote and promastigote forms of $L$. donovani.
\end{tabular}

\section{INTRODUCTION}

Leishmaniasis is a major public health problem caused by an intracellular obligate protozoan parasite of the genus Leishmania. Leishmaniasis, by its various clinical forms, causes significant morbidity and mortality in the developing countries of the world every year. It is estimated that 0.2 to 0.4 million new cases of visceral leishmaniasis, 0.7 to 1.3 million new cases of cutaneous leishmaniasis with 20,000 to 30,000 deaths occur every year worldwide (Rama et al., 2015). Despite the severity of this parasitic disease, till date, there is no vaccine available to efficiently prevent or cure leishmaniasis. The currently used chemotherapy has unpleasant side effects such as toxicity, variable efficiency

\section{${ }^{*}$ Corresponding Author}

Arushdeep Sidana, Assistant Professor Microbiology

Department of Biological and Paramedical Sciences, RIMT University,

Punjab, India.E-mail: arush.sidana@gmail.com between different species and development of drug resistance in the parasite (Camacho et al., 2003; Croft et al., 2006). People living in rural areas of the developing countries are still dependent on traditional medicines to heal their ailments (Chan-Bacab and Pena-Rodriguez, 2001). There is a need to explore the potential of natural products obtained from the plants used by traditional healers which may eliminate the parasite from the host without causing any side effects. Several plant species have been screened for leishmanicidal activity after extraction with different solvents like petroleum ether, chloroform, hexane, ethyl acetate, methylene chloride, methanol and tested in vitro and in vivo against Leishmania parasite (Fournet et al., 1996; Ghosh et al., 2011; Pereira et al., 2010).

The discovery and development of leishmanicidal biomolecules is also aided by the biochemical studies of the metabolic pathways of the parasite. In this approach, the enzymes essential for the survival of Leishmania are identified 
and targeted by lead molecules (Ogungbe and Setzer, 2013). Some known drug targets of $L$. donovani which are essential for the survival of the parasite and may be employed for in silico studies include adenylosuccinate lyase which converts adenylosuccinate to AMP and fumarate, an important step in purine salvage, Cyclophilin and $N$-myristoyl transferase are involved in co-/post-translational protein processing. Dihydroorotate dehydrogenase is involved in de novo pyrimidine biosynthetic pathway and pteridine reductase 1 is responsible for the salvage of pteridines (Ogungbe and Setzer, 2013; Boitz et al., 2013; Sahi et al., 2012).

Gentiana kurroo is a critically endangered medicinal plant belonging to the family Gentianaceae. It is mainly found in the North-Western Himalayas on south-facing steeper slopes along dry and rocky grasslands (Khuroo et al., 2005). In traditional medicine, the roots and rhizomes of G. kurroo are used for the treatment of anorexia, bronchial asthma, gastric infections, leucoderma and urinary tract infections. It is also used as anthelmintic, astringent, antipsychotic, sedative, stomachic and carminative (Raina et al., 2003).

The present study was aimed to assess the antileishmanial potential of methanolic root extract of $G$. kurroo in vitro against $L$. donovani followed by in silico docking of the reported phyto-constituents present in the methanolic root extract with five known drug targets of $L$. donovani to recognize the compound(s) responsible for the antileishmanial activity.

\section{MATERIALS AND METHODS}

\section{In vitro assessment of antileishmanial activity}

\section{Preparation of extracts}

Authenticated plants of G. kurroo were obtained from Plant Tissue Culture Laboratory, Department of Biotechnology, Shoolini University, Solan, H.P., India. The roots of the plants were thoroughly washed with tap water and subsequently with distilled water and air dried at room temperature. After complete drying, the roots were ground to yield a coarse powder. The powdered roots were extracted with methanol by Soxhlet extraction method for 24 hours. The extract was concentrated under reduced pressure using rotary evaporator. The dried extract was then stored in an air tight bottle at $4^{\circ} \mathrm{C}$ until use.

\section{Parasite stock culture}

The Axenic culture of L. donovani (LdMIPL-1) was maintained at $25^{\circ} \mathrm{C}$ in RPMI 1640 (Himedia) medium supplemented with $10 \%$ heat inactivated Fetal Bovine Serum (FBS, Himedia), streptomycin $(150 \mu \mathrm{g} / \mathrm{ml})$, penicillin $\mathrm{G}(100$ $\mu \mathrm{g} / \mathrm{ml})$ and gentamycin $(150 \mu \mathrm{g} / \mathrm{ml})$ at $\mathrm{pH} 7.2$.

\section{Antileishmanial assay}

For antileishmanial activity, promastigotes of $L$. donovani were sub-cultured in Schneider's Insect Medium (Himedia) supplemented with $10 \%$ heat inactivated FBS, streptomycin $(150 \mu \mathrm{g} / \mathrm{ml})$, penicillin $\mathrm{G}(100 \mu \mathrm{g} / \mathrm{ml})$ and gentamycin $(150 \mu \mathrm{g} / \mathrm{ml})$. The antileishmanial screening was performed in 96-well flat bottom tissue culture plates (Corning Life Sciences, USA). One hundred microliters of cell suspension containing $2 \times 10^{6}$ to $3 \times 10^{6}$ cells $/ \mathrm{ml}$ was poured in each well of the plate. Four different concentrations of the methanolic root extract i.e. $100,250,350$ and 500 $\mu \mathrm{g} / \mathrm{ml}$ dissolved in dimethyl sulfoxide $(<0.025 \% \mathrm{v} / \mathrm{v})$ were added to the culture. The plates were then incubated at $25^{\circ} \mathrm{C}$ for 24-48 hours. Sodium stibogluconate was used as positive controls and cell suspension with $0.025 \%$ DMSO was used as a negative control. The inhibition of the promastigotes was assessed by measuring the cleavage of $10 \mathrm{mg} / \mathrm{ml}$ of MTT \{3-(4.5-dimethylthiazol-2-yl)-2.5-diphenyl tetrazolium bromide $\}$ (Mossman, 1983). The absorbance was measured by using ELISA plate reader (BioTek, USA) at $595 \mathrm{~nm}$. The percent growth inhibition was calculated by the following formula:

$$
\% \text { of inhibition }=\frac{O D \text { control }- \text { OD treated }}{\text { OD control }} \times 100
$$

\section{Statistical analysis}

The antileishmanial assay was performed in triplicate with three replicates of each concentration tested. The results were expressed as mean \pm standard error of mean. The overall variation in a set of data was analysed by one way analysis of variance (ANOVA). A value of $\mathrm{P}<0.05$ was considered significant.

\section{In silico study}

\section{Selection and preparation of drug targets for docking}

A total of five L. donovani proteins known as efficient drug targets were selected for docking (Table 1). Pdb files of 3D structures of all the drug targets were downloaded from Protein Data Bank and all the non-protein molecules (hetero-atoms) were removed. By using AutoDockTools-1.5.6, pdb files of all the receptors were modified by adding polar hydrogens and converted to pdbqt files which is an extended pdb format used for molecular docking analysis.

Table 1: List of $L$. donovani drug targets used for molecular docking analysis.

\begin{tabular}{lcc}
\hline Drug target & Abbreviation & Accession number \\
\hline$N$-myristoyl transferase & LdonNMT & PDB (2WUU) \\
Cyclophilin & LdonCyp & PDB (3EOV) \\
Dihydroorotate dehydrogenase & LdonDHODH & PDB (3C61) \\
Adenylosuccinate lyase & LdonASL & PDB (4MX2) \\
Pteridine reductase 1 & LdonPTR1 & PDB (2XOX) \\
\hline
\end{tabular}

\section{Ligand preparation}

A total of thirteen reported phytochemical constituents found in the roots of G. kurroo (Figure 1) were drawn using ChemDraw Ultra 10.0 software (Maurya et al., 2012; Wani et al., 2013). Smiles of all the structures were converted into pdb files using online smiles converter (http://cactus.nci. nih.gov/services/translate/). Pdb files were then optimised and converted to pdbqt files for molecular docking by using AutoDock Tools 1.5.6. 
<smiles>C=CC1C2=CCOC(=O)C2=CO[C@@H]1O[C@@H]1OC2C(O)C3OC(CO)C(O3)C21</smiles>

A) Gentiopicroside

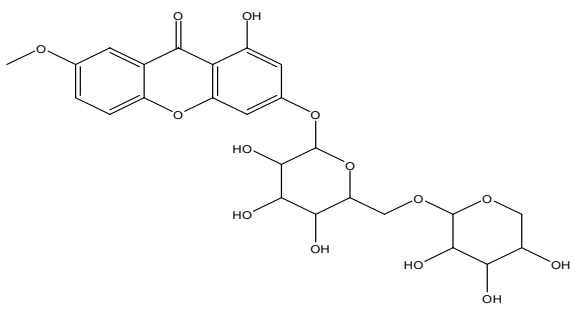

D) Gentioside

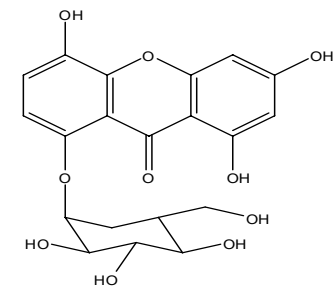

G) Norswertianolin<smiles>C=CC1C2OC(CO)C(O)C2C(O)C2O[C@@H]3OC=C4C(=O)OCC[C@]4(O)C3C12O</smiles>

J) Swertiamarin<smiles>O=C1c2cc(O)ccc2Cc2cc(O[O-])cc(O)c21</smiles>

B) Gentisin

E) Isogentisin

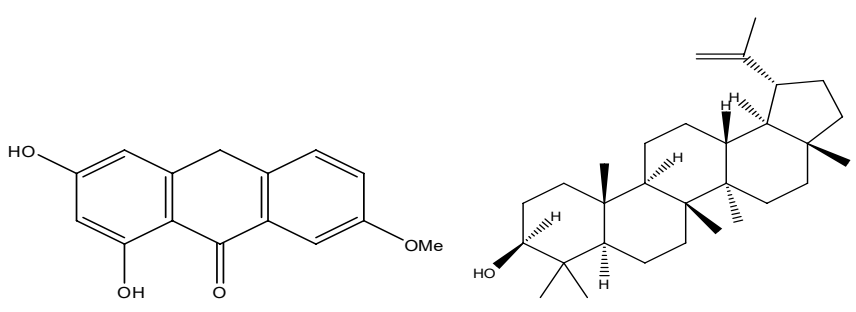

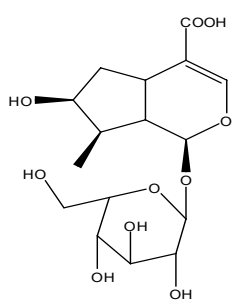

C) Loganic acid

F) Lupeol

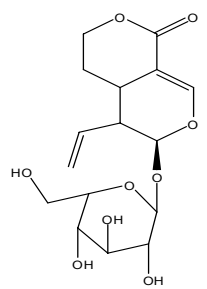

I) Sweroside

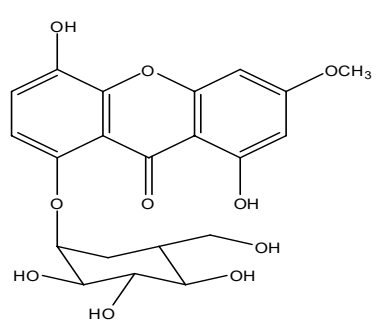

K) Swertianolin

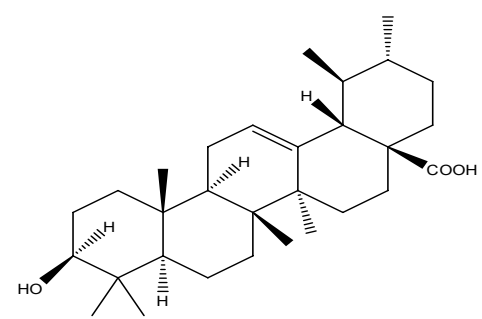

L) Ursolic acid<smiles>COc1cc2oc(-c3ccc(O)cc3)cc(=O)c2c(O)c1C1OC2(O)C(O)C(O)C1C2O</smiles>

M) Swertisin

Fig. 1: Molecular structures of the phyto-constituents found in methanolic root extract of G. kurroo (A-M) 


\section{Molecular docking analysis}

Molecular docking analysis was carried out to evaluate the efficacy of phyto-constituents found in the roots of G. kurroo against five drug targets which are essentially required for the survival of $L$. donovani by using AutoDock Vina with default parameters (Trott and Olson, 2010). The known active sites of various drug targets for inhibitors and substrates were identified using "Ligand Explorer" tool in Protein Data Bank (Barrack et al., 2011; Brannigan et al., 2010; Venugopal et al., 2009).

\section{RESULTS}

\section{In vitro antileishmanial activity}

Methanolic root extract of G. kurroo was screened for antileishmanial activity against promastigote forms of $L$. donovani. The mortality rate of $L$. donovani was assessed by using MTT reduction assay. Maximum inhibition (43.1\%) of the parasite was observed at the concentration of $500 \mu \mathrm{g} / \mathrm{ml}$; followed by $41.68 \%$, $40.49 \%$, and $37.51 \%$ inhibition with concentrations of 350,250 and $100 \mu \mathrm{g} / \mathrm{ml}$, respectively (Figure 2). The antileishmanial activity of G. kurroo root extract was considerably low as compared to the sodium stibogluconate which was used as standard drug against $L$. donovani (Figure 3).

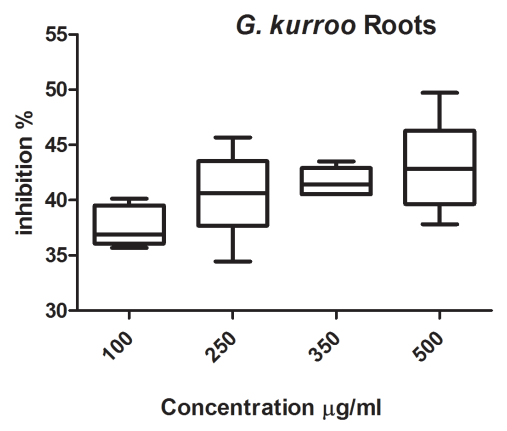

Fig. 2: Percent inhibition of the $L$. donovani promastigotes after 24 hours of incubation with four different concentrations of methanolic root extract of $G$. kurroo. The standard error of the mean was calculated as $0.731,1.554,0.502$, and 1.685 in case of $100,250,350$ and $500 \mu \mathrm{g} / \mathrm{ml}$, respectively with a $P$ value of 0.0279 .

\section{In silico docking analysis}

In silico analysis by molecular docking of the phytoconstituents in the methanolic root extract of G. kurroo with five known protein targets of $L$. donovani was carried out to identify the compound(s) responsible for the leishmanicidal activity. The resultant receptor-ligand complexes were generated using PyMol Molecular Graphic System to know whether the ligands were bound to the amino acid residues of the active site or elsewhere in the receptor (Figure 4).

It was observed that all the phyto-constituents found in methanolic root extract of G. kurroo have considerably low binding energies against all the five $L$. donovani drug targets (Table 2). Gentioside and norswertianolin exhibited minimum binding energies with most of the drug targets used. Out of these two only gentioside was bound to the active site of the four protein targets i.e. LdonASL, LdonCyp, LdonNMT and LdonPTR1.
Norswertianolin was bound only to the active site of LdonDHODH whereas; the binding was random for the other four receptors and somewhat away from the active site. The active site amino acid residues of all the five drug targets to which the best ligands were bound are enlisted in Table 3. LdonASL and LdonNMT are the two proteins which have shown very low binding energies with most of the phyto-constituents but their active sites were properly blocked only by gentioside. The binding modes of each ligand may be different for all the drug targets used as the active sites of all the targets possess characteristic amino acid residues which make them unique. Therefore, the gentioside was interacted with different amino acid residues in all the drug targets (Figure 4a, $4 \mathrm{~b}, 4 \mathrm{~d}, 4 \mathrm{e})$. The in silico study revealed that gentioside may be responsible for the antileishmanial activity shown by the methanolic root extract of G. kurroo.

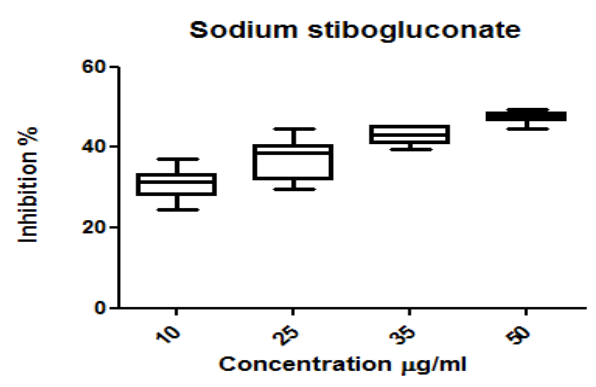

Fig. 3: Percent inhibition of the L. donovani promastigotes after 24 hours of incubation with four different concentrations $\left(1 / 10^{\text {th }}\right.$ of $G$. kurroo extract) of standard drug (sodium stibogluconate).

\section{DISCUSSION}

Leishmaniasis, being endemic in developing countries, establishes a worldwide health problem. Control of the disease becomes more difficult due to lack of an effective vaccine and some safe chemotherapeutic agents. The current chemotherapy of leishmaniasis includes pentavalent antimonials, liposomal formulations of amphotericin B, pentamidine and miltifosine and all of these are associated with one or more drawbacks which limit their use (Santos et al., 2008). In the search of some new leishmaniasis control agent research is going on worldwide and is more concentrated towards the use of natural products rather than synthetic chemicals. Biological molecules extracted from plant and marine natural products represent an important source of novel therapeutics against various forms of leishmaniasis (Rocha et al., 2005; Tempone et al., 2011).

G. kurroo is used in ethno-medicine for the treatment of several diseases since ancient times. The methanolic root extract of G. kurroo is known to have potential anticancer activity by inducing apoptosis in human pancreatic cancer cell line (MiaPaCa-2) through the disruption of mitochondrial membrane potential (Wani et al., 2013). Lupeol and ursolic acid which are found in the methanolic root extract of $G$. kurroo possess significant immune-modulatory activity (Maurya et al., 2012). Methanolic whole plant extract has been reported to possess anti-inflammatory and immunosuppressant activities (Khan et al., 2014). G. kurroo has not been studied so far for its antileishmanial activity.

In the present study methanolic extract of $G$. kurroo roots was evaluated for its antileishmanial activity against $L$. donovani. 
We have observed $43.1 \%$ inhibition with a concentration of $500 \mu \mathrm{g} /$ $\mathrm{ml}$. The findings of the study are in accordance with the previously reported studies on Artemisia aucheri and Camellia sinensis where the methanolic extracts of these plants at concentrations of $600-750 \mu \mathrm{g} / \mathrm{ml}$ showed significant antileishmanial activity against both amastigote and promastigote forms of L. major (Sharif et al., 2006). In another study, it was reported that methanolic extract of Alstonia scholaris at a concentration of $500 \mu \mathrm{g} / \mathrm{ml}$ showed an inhibition of $40.3 \%$ inhibition of $L$. donovani promastigotes (Sidana and Farooq, 2015). The activity shown by methanolic extract of G. kurroo in the present study was considered significant as the plants used for extraction were in vitro cultivated and were not obtained from their natural habitat.

\begin{tabular}{lccccc} 
Table 2: Binding energies of the 13 phyto-constituents found in the methanolic root extract of G. kurroo against five $L$. donovani drug targets (kcal/m \\
\hline Receptors -> Ligands & LdonASL & LdonCyp & LdonDHODH & LdonNMT & LdonPTR1 \\
\hline Gentiopicroside & -8.1 & -7.0 & -6.8 & -8.4 & -8.5 \\
Gentioside & -10.4 & -8.8 & -8.0 & -10.3 & -8.9 \\
Gentisin & -8.3 & -7.4 & -7.3 & -9.7 & -8.0 \\
Isogentisin & -8.6 & -7.7 & -8.0 & -9.7 & -8.2 \\
Loganic acid & -7.8 & -7.3 & -7.0 & -8.9 & -8.0 \\
Lupeol & -9.4 & -6.6 & -7.6 & -9.3 & -7.4 \\
Norswertianolin & -8.8 & -8.1 & -9.6 & -10.4 & -8.6 \\
Oleanolic acid & -10.2 & -6.6 & -8.2 & -8.7 & -7.6 \\
Sweroside & -8.6 & -7.2 & -7.0 & -8.0 & -7.8 \\
Swertiamarin & -8.7 & -7.0 & -7.3 & -10.1 & -7.6 \\
Swertianolin & -9.2 & -8.2 & -7.3 & -10.2 & -8.7 \\
Swertisin & -9.4 & -7.2 & -8.0 & -9.8 & -7.5 \\
Ursolic acid & -9.3 & -7.0 & & &
\end{tabular}

Table 3: List of amino acid residues of the active sites of drug targets to which the used ligands were bound.

\begin{tabular}{ccc}
\hline Serial Number & Drug Target & Amino acid residues in active sites \\
\hline 1 & $N$-myristoyl transferase & Leu169, Val171 \\
2 & Cyclophilin & Arg78, Gln86 \\
3 & Dihydroorotate dehydrogenase & Leu72, Lys 44, Asn195, Ser 196, Asn68, Gly272 \\
4 & Adenylosuccinate lyase & Gln-273, Thr-148, Ser-149 \\
5 & Pteridine reductase 1 & Ser40 \\
\hline
\end{tabular}

In earlier studies, thirteen phyto-constituents have been reported in the methanolic root extract of G. kurroo. These suspected phyto-constituents must be validated and voted up for further in vitro and in vivo antileishmanial assays to minimize the expenses and time consumption. This may be achieved by molecular docking analysis of the phyto-constituents with potential drug targets. Virtual screening is a useful method for the preliminary screening of the drugs against a particular pathogen as it requires less time and resources. It is extensively used for screening of synthetic compounds as well as natural products against Echinococcus, Plasmodium, Mycobacterium and other tropical infectious agents (Crowther et al., 2010; Mehlin, 2005; Prakash et al., 2010; Sundaramurthi et al., 2011).

In this study, we have targeted five L. donovani drug targets for the preliminary screening of thirteen reported phytoconstituents by in silico protein-ligand docking to find out the compound responsible for in vitro antileishmanial activity and to validate it for further experiments. In molecular docking, we observed that gentioside was able to block the active sites of four out of five drug targets used and may play an important role in the inhibition of their catalytic activity which may lead to interruption of various important metabolic pathways of $L$. donovani. On the other hand, norswertianolin, which has shown minimum binding energy for LdonDHODH and blocked its active site, may cause interruption in de novo pyrimidine biosynthesis in the parasite thereby inhibiting the parasite growth and multiplication. The remaining eleven compounds have shown low binding energies with most of the drug targets used; however, those compounds were binding to the sites away from the known active sites of the drug targets.

\section{CONCLUSION}

The findings of the present study suggested that methanolic root extract of $G$. kurroo possess significant antileishmanial activity against the $L$. donovani promastigotes. In silico docking revealed that both gentioside and norswertianolin may be the major players responsible for the in vitro antileishmanial activity of methanolic root extract of G. kurroo. These two compounds have never been tested before for their antileishmanial potential. Thus, further studies are directed at purification of these two compounds and their screening for antileishmanial activity against amastigote and promastigote forms of $L$. donovani. 

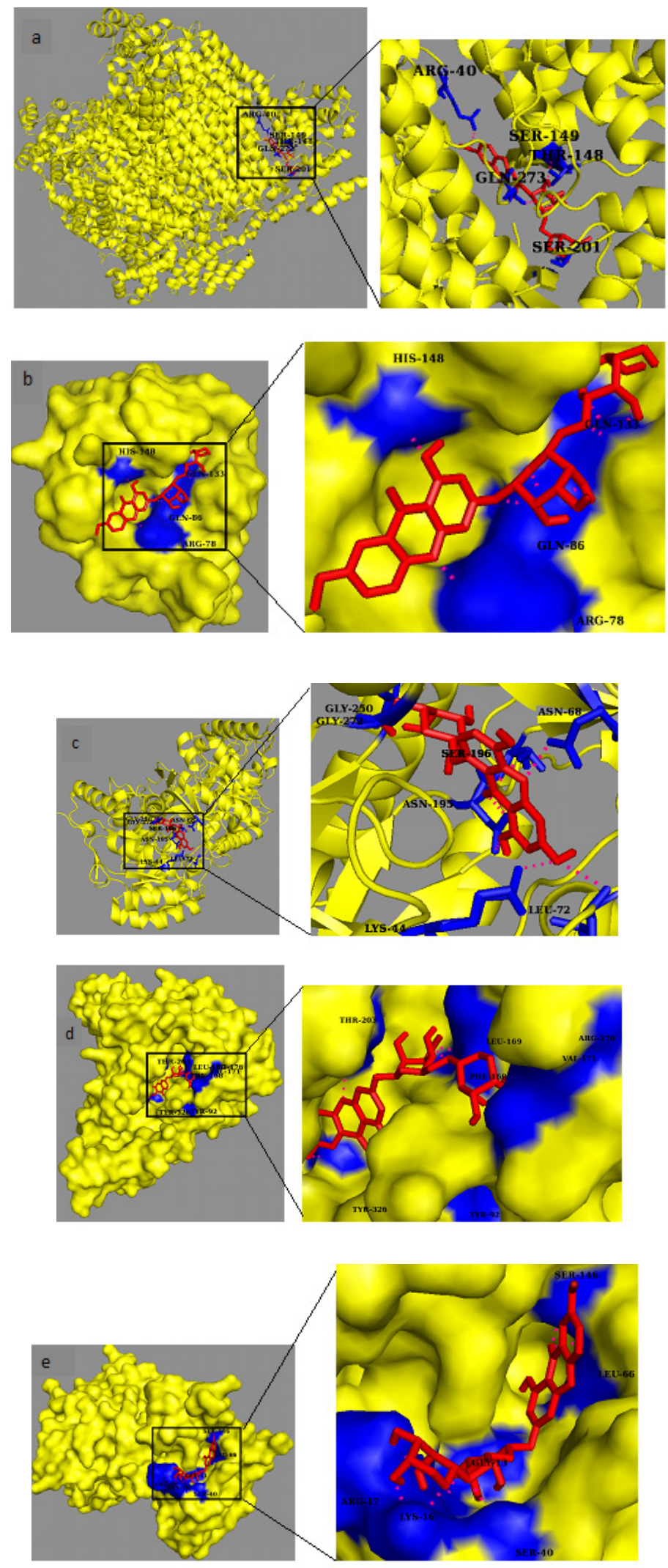

Fig. 4: Ligand-receptor complex models of best docked ligands (phyto-constituents in root extract of G. kurroo) showing the interaction of ligands in the known active sites of five receptors (a) LdonASL with Gentioside, (b) LdonCyp with gentioside, (c) LdonDHODH with norswertianolin, (d) LdonNMT with gentioside and (e) LdonPTR1 with gentioside. Here, ligand is shown in red, yellow is the surface of drug target, blue are the active amino acid residues to which the ligand is bound and pink dotted lines are the hydrogen bonds between ligand and amino acid residues. 


\section{ACKNOWLEDGEMENT}

Authors are thankful to Shoolini University, Solan, H.P., India for providing the required facilities and resources to carry out this research work.

\section{REFERENCES}

Barrack KL, Tulloch LB, Burke LA, Fyfe PK, Hunter WN. Structure of recombinant Leishmania donovani pteridine reductase reveals a disordered active site. Acta Crystallogr Sect F Struct Biol Cryst Commun. 2011;67(1):33-37.

Boitz JM, Strasser R, Yates PA, Jardim A, Ullman B. Adenylosuccinate synthetase and adenylosuccinate lyase deficiencies trigger growth and infectivity deficits in Leishmania donovani. J Biol Chem. 2013;288(13):8977-8990.

Brannigan JA, Smith BA, Yu Z, Brzozowski AM, Hodgkinson MR, Maroof A. N-myristoyl transferase from Leishmania donovani: Structural and functional characterisation of a potential drug target for visceral leishmaniasis. J Mol Biol. 2010;396(4):985-99.

Camacho Md, Phillipson JD, Croft SL, Solis PN, Marshall SJ, Ghazanfar SA. Screening of plant extracts for antiprotozoal and cytotoxic activities. J Ethnopharmacol. 2003;89(2-3):185-191.

Chan-Bacab MJ, Pena-Rodriguez LM. Plant natural products with leishmanicidal activity. Nat Prod Rep. 2001;18(6):674-688.

Croft SL, Sundar S, Fairlamb AH. Drug resistance in leishmaniasis. Clin Microbiol Rev. 2006;19(1):111-126.

Crowther GJ, Shanmugam D, Carmona SJ, Doyle MA, Hertz-Fowler C, Berriman M. Identification of attractive drug targets in neglected-disease pathogens using an in silico approach. PLoS Negl Trop Dis. 2010;4(8):e804.

Fournet A, Ferreira ME, Rojas De Arias A, Fuentes S, Torres S, Inchausti A. In vitro and in vivo leishmanicidal studies of Peperomia galioides (Piperaceae). Phytomedicine. 1996;3(3):271-275.

Ghosh S, Debnath S, Hazra S, Hartun A, Thomale K, Schultheis M. Valeriana wallichii root extracts and fractions with activity against Leishmania spp. Parasitol Res. 2011;108(4):861-871.

Khan M, Khalid G, Bashir AG, Seema A, Akhtar HM, Akbar M. Scientific validation of Gentiana kurroo Royle for anti-inflammatory and immune-modulatory potential. ISRN inflamm. 2014; 701765.

Khuroo AA, Dar GH, Khan ZS, Reshi ZA. Observations on Gentiana kurroo Royle, a critically endangered medicinal plant from the Kashmir Himalaya, India. Endangered species update. 2005;22:139-143.

Maurya A, Khan F, Bawankule DU, Yadav DK, Srivastava SK. QSAR, docking and in vivo studies for immuno-modulatory activity of isolated tri-terpenoids from Eucalyptus tereticornis and Gentiana kurroo. Eur J Pharm Sci. 2012;47(1):152-61.

Mehlin C. Structure-based drug discovery for Plasmodium falciparum. Comb Chem High Throughput Screen. 2005;8(1):5-14.

Mossman T. Rapid colorimetric assay for cellular growth and survival: Application to proliferation and cytotoxicity assays. J Immunol Methods. 1983;65(1-2):55-63.

Ogungbe IV, Setzer WN. In-silico Leishmania target selectivity of antiparasitic terpenoids. Molecules. 2013;18(7):7761-7847.

Pereira IO, Marques MJ, Pavan ALR, Codonho BS, Barbieri CL, Beijo LA. Leishmanicidal activity of benzophenones and extracts from Garcinia brasiliensis Mart. fruits. Phytomedicine. 2010;17(5):339-345.

Prakash N, Patel S, Faldu NJ, Ranjan R, Sudheer DVN. Molecular docking studies of antimalarial drugs for malaria. J Comput Sci Syst Biol. 2010;3:70-73.

Raina R, Behera MC, Chand R, Sharma Y. Reproductive biology of Gentiana kurroo Royle. Curr Sci. 2003;85(5):667-670.

Rama M, Kumar NV, Balaji S. A comprehensive review of patented antileishmanial agents. Pharm Pat Anal. 2015;4(1):37-56.

Rocha LG, Almeida JR, Macêdo RO, Barbosa-Filho JM. A review of natural products with antileishmanial activity. Phytomedicine. 2005;12(6-7):514-35.

Sahi S, Tewatia P, Ghosal S. Leishmania donovani pteridine reductase 1: comparative protein modelling and protein-ligand interaction studies of the leishmanicidal constituents isolated from the fruits of Piper longum. J Mol Model. 2012;18(12):5065-5073.

Santos DO, Coutinho CE, Madeira MF, Bottino CG, Vieira RT, Nascimento SB. Leishmaniasis treatment - A challenge that remains: A review. Parasitol Res. 2008;103(1):1-10.

Sharif M, Ziaei H, Azadbakht M, Daryani A, Ebadattalab A, Rostami M. Effect of Methanolic Extracts of Artemisia aucheri and Camellia sinensis on Leishmania major (In Vitro). Turk J Med Sci. 2006;36(6):365369 .

Sidana A, Farooq U. Evaluation of antileishmanial activity of plants used in Indian traditional medicine. Bangladesh J Pharmacol. 2015; 10:423-426.

Sundaramurthi JC, Kumar S, Silambuchelvi K, Hanna LE. Molecular docking of azole drugs and their analogs on CYP121 of Mycobacterium tuberculosis. Bioinformation. 2011;7(3):130-133.

Tempone AG, Martins de Oliveira C, Berlinck RG. Current approaches to discover marine antileishmanial natural products. Planta Med. 2011;77(6):572-85.

Trott O, Olson AJ. AutoDock Vina: Improving the speed and accuracy of docking with a new scoring function, efficient optimization and multithreading. J Comput Chem. 2010;31(2):455-461.

Venugopal V, Datta AK, Bhattacharyya D, Dasgupta D, Banerjee R. Structure of cyclophilin from Leishmania donovani bound to cyclosporin at $2.6 \AA$ resolution: Correlation between structure and thermodynamic data. Acta Crystallogr Sect D Biol Crystallogr. 2009; 65: 1187-1195.

Wani BA, Ramamoorthy D, Rather MA, Arumugam N, Qazi $\mathrm{AK}$, Majeed R. Induction of apoptosis in human pancreatic MiaPaCa-2 cells through the loss of mitochondrial membrane potential $(\Delta \Psi \mathrm{m})$ by Gentiana kurroo root extract and LC-ESI-MS analysis of its principal constituents. Phytomedicine. 2013;20(8-9):723-33.

\section{How to cite this article:}

Sidana A, Kaushal S, Farooq U. Evaluation of antileishmanial potential of Gentiana kurroo Royle by in vitro and in silico methods. J App Pharm Sci, 2018; 8(02): 143-149. 